\title{
Integrating Practical and Epistemic Actors: Co- Constructing a Knowledge Organization System to Address Housing Insecurity in West Philadelphia
}

\author{
Samantha Stein \\ University of Pennsylvania \\ swstein@sas.upenn.edu
}

\author{
Diana Susan Nicholas \\ Drexel University \\ dsn35@drexel.edu
}

\author{
Yvonne Michael \\ Drexel University \\ ylm23@drexel.edu
}

\author{
Thanh My Nguyen \\ Drexel University alum \\ thanhmynguyen62@gmail.com
}

\author{
Kristen Giordano \\ Drexel University \\ krg66@drexel.edu
}

\begin{abstract}
In response to colonial legacies of divisiveness and paternalism underpinning the development of knowledge organization systems (KOS) and thus impeding their appeal, accessibility, and usefulness to diverse stakeholders (Castleden, Morgan, \& Lamb, 2012), this case study explores the challenges and opportunities inherent to the design of a malleable, sustainable KOS as part of an mHealth tool called Map the Gap. Map the Gap intends to reduce the burden of housing insecurity in West Philadelphia. By examining the active cultivation of communal ties between the "epistemic" and "practical" actors (Callon, 4, 2004) who substantiate Map the Gap, as well as the sociotechnical infrastructure which shapes and is shaped by such ties, the processes of collaboration underpinning functionality decisions are delineated. This paper reflects on the way KOS sociotechnical structures defy and challenge traditional academic and community models of research and development, thus requiring a unique, temporally-conscious embracement of select and dynamic collaborations. By elucidating and evaluating the considerations and practices central to Map the Gap, we seek to yield a template for cultivating healthy KOS sociotechnical structures.
\end{abstract}

\section{Keywords}

Actor-network theory, dependent origination, socio-technical structures, politics of artifacts, urban design, humancentered design, co-construction, participant-action framework, knowledge organization system, housing insecurity, culture of health, design research, information ecology, democratic innovation, participatory design, communitybased participatory research

\section{INTRODUCTION}

In 2017, Philadelphia pledged $\$ 500,000$ towards mitigating city-wide evictions via low-cost legal assistance provided by Community Legal Services (CLS) (Blumgart, 2018). This measure is intended to boost tenant-representation in small claims court from the current five to eight percent, up to ten percent (Blumgart, 2018). Given the scarcity of this

$81^{\text {st }}$ Annual Meeting of the Association for Information Science \& Technology | Vancouver, Canada | Nov. 10 - 14, 2018

Author(s) Retain Copyright legal resource, referrals to CLS must be made carefully.

As data scientists and designers for Map the Gap, an in-development mobile health solution to housing insecurity in West Philadelphia, 'we' are tasked with creating an algorithm that determines which intervention users receive referrals to Community Legal Services (CLS). While ideally all users with legal needs would receive such referrals, this would overwhelm the system's capacity, reducing its efficiency and efficacy. Thus, 'we' are forced to prioritize: Does immediate duress, long term need, or likelihood to win in court warrant the most weight? How should each of these factors be assessed?

In the above example, the "we" granted power to prioritize life via authorship of a knowledge organization system (KOS) alludes to a particular set of persons embodying particular roles and bringing to those roles a particular set of "unknown knowns," "the disavowed beliefs, suppositions, and obscene practices ... [that] form the background of our public values." (Zizek, 137, 2006). By considering the material presentation of a KOS to be a manifestation of "unknown knowns" (Zizek, 137, 2006) or subconsciously situated biases, the need for diversity amongst developers is elucidated. Such diversity of authorial voice is supported by a participant action/human-centric model of research and design. This paper explores the challenges and opportunities inherent to designing a malleable, sustainable KOS as part of an mHealth tool to address housing insecurity in West Philadelphia by examining the active cultivation of communal ties between the "epistemic" actors, who "aim primarily to produce formal knowledge" and "practical" actors, who "focus on solving technical problems and developing know-how" (Callon, 4, 2004). These actors substantiate such a KOS, as well as the sociotechnical infrastructure which shapes and is shaped by such ties.

The CLS dilemma is just one of many ethical issues embedded in the process of designing a digital KOS as part of a tool, Map the Gap, that will help Philadelphians (and eventually people nationwide) to live healthier lives in the midst of epidemic housing insecurity. Ultimately, Map the Gap will connect tenants, landlords, community-based organizations, government agencies, and other resources in Philadel- 
phia by 1) providing personalized navigation of Philadelphia's housing system, 2) facilitating communication between parties, and 3) enabling service exchange (a local currency). While the expected functionality of Map the Gap is noteworthy, it is the structured malleability of such functionality and the processes of collaboration underpinning functionality decisions which yield a template for cultivating healthy KOS sociotechnical structures.

\section{STRUCTURALLY EMBEDDED POLITICS AS UNKNOWN KNOWNS}

Value judgments are oft-overlooked innate, political components of algorithms and artifacts (Winner, 1980), such as those integral to Map the Gap; the language (ie. jargonistic, passive, assertive, localized, etc.), timing (hypothetical, preventative, interventional, reflective), and direction of the communications (ie. landlord to tenant, tenant to landlord, tenant to councilwoman, etc.), as well as the services (ie. roofing, plumbing, landlord background checks, legal assistance, etc.) and servicers (brands, agencies, organizations, private contractors, etc.) promoted by the system reflect the subconscious biases/values of the authorial team. Thus, it is imperative that the development of such algorithms/artifacts and the values they institutionalize occur inclusive of local stakeholders. While the inclusion of historically underrepresented stakeholders in the design process of community-oriented KOS's is often presumed to be financially and temporally taxing (Abras, Maloney-Krichmar, \& Preece, 2004), the democratization of the design process is critical for product feasibility and sustainability (Preece, Rogers, \& Sharp, 2002), as well as ethically essential (Bjorgvinsson, Ehn, \& Hillgren, 2010; Janack, 137, 1997).

\section{CHALLENGES OF CREATING BROADLY ACCEPTED KOS WHICH INTEGRATE PRACTICAL AND EPISTEMIC COMMUNITIES}

Although ethically obligatory and practically useful, integrating practical and epistemic communities proves challenging when collaboratively produced outcomes must be widely legitimized. Within the historic environs Map the Gap sits at the edge of, a socially constructed dichotomy exists wherein academic actors maintain both epistemic and practical privilege, despite lacking practical authority (the ability to successfully implement programs/systems) with regards to local interventions (Janack, 130, 1997). Local actors, despite their practical authority (conferred by their revered status amongst non-academic populations), have been denied practical privilege. Instead they historically have been relegated to the sidelines of research and development, forced to serve as subjects rather than embraced as drivers of collaborative innovation. Out of this dichotomy between academic and local actors emerges a mutual exclusivity which renders integrated outcomes difficult to obtain and subject to harsh scrutiny; integration challenges the fundamentals upon which authority is constructed. The authority to produce formal knowledge is granted and moderated by those who tarry with such formal knowledge themselves, affirming, negating, and augmenting it to form a highly exclusive 'citation culture' (Todd \& Ladle, 2008). Such a culture, historically (Janack, 132, 1997) and presently (Koedel, 2017), is dominated by wealthy, white males. Statistically speaking, to be poor, of color, or female is to be minimally immersed in a culture of methodological peer review, or in other words to lack epistemic privilege and thus be denied of epistemic authority (Janack, 1997).

In the case of Map the Gap, the actors with automatic (socially conferred) epistemic privilege are entirely white and upper-middle income, albeit female. The intended users, primarily black, low-income females, who harbor practical authority lack the epistemic privilege to translate their unique knowledge into academically legitimized knowledge and action. Thus the team strives to create sociotechnical structures (to be detailed) which evoke and contextualize the voices of non-privileged epistemic actors, as is needed to integrate diverse epistemological contributions to the KOS for an end of a bipartisan or widely accessible, useful KOS.

\section{INDIVIDUAL/INSTITUTIONAL IDENTITY CONFLICTS WHICH MOTIVATE KOS DESIGN}

As researchers with both institutional and individual identities we must navigate the tensions associated with creating a KOS that is simultaneously sustainable, feasible, and ethical with respect to local users, as well as academically accepted. To obtain such comprehensive validity given that the aforementioned characteristics are oftentimes mutually exclusive, we must weigh means and ends that should not be at odds in a healthy system, putting ourselves and our peers at stake. While our research relies on institutional faculties for sustenance (funding, overhead, faculty support, public backing, etc.), the end goal of our science is humancentered; accordingly, we traverse a space in which our personal values, and the means to realizing our values as ends are misaligned.

While universities are in theory service-based, practically they are economically operational entities. The University of Pennsylvania Board of Trustees' Vice President for Government and Community Affairs Jeff Cooper attests to such a dichotomy, claiming, "We're not a social service agency — we're a university" (Washington \& Wright, 2014). Thus, two primary tensions emerge: 1) Misalignments in values and practices needed for sustaining the project/institution and 2) Harsh realities of integrating expertise in the context of hierarchical, evidence-based norms of operation.

\section{Misalignments in Values and Practices Needed for Sus- taining the Project/Institution}

Our research is financially supported by the university, and thus to preserve the institutions' epistemic authority we are pressured to collect data sets that fulfill academia's conceptualization of research benefit. Benefit is operationalized through the publication of results in journals which are typically inaccessible to community members. Given the scarcity of time, the use of community member time to collect data sets deemed significant solely by academia seems inappropriate and maleficent in that it imparts no direct benefit to said community members, and deprives them of time. Additionally, to preserve university holding in communities, it seems most institutionally loyal to promote university-sponsored services to the community, even when they are not most fitting to the community's needs nor conducive to a healthy local economy. To stray from university loyalties could jeopardize the future of the project as a whole, yet to maintain such loyalties renders only subpar, 
and thus inadequate, benefit to communities.

\section{Harsh Realities of Integrating Expertise in the Context of Hierarchical, Evidence-Based Norms of Operation} The social construction of cultural capital renders local community members excluded from the realm of relevance when assessing the value of academic pursuits. Numerical assessments are oft prioritized over locals' anecdotal understandings of their own communities. In addition, tense racial dynamics and difficulty dealing with illiteracy leave locals further removed from participation in research. However, value to the community dissipates and rifts between the community and institution deepen when the community is excluded from product development.

For example, misalignments in language used by epistemological communities and practical communities/users can leave products inaccessible to those they are intended for. At one community co-design session, the Map the Gap team learned that attendees from local communities considered themselves to be middle class because they did not qualify for welfare and other benefits (due to niche clauses in policy), despite having income levels consistent with academia's conceptualization of lower-class. Had we not identified such a terminological variation, the language of the KOS in-design would be less accessible.

While the functional integration of such specific terminology is notable, the broader practices which underpin Map the Gap's sociotechnical structures allowing for such terminological variations to be illuminated and integrated in a dynamic matter on a large scale are of greater interest when developing a template for healthy KOS sociotechnical structures.

\section{A PRACTICAL CULTURE OF EPISTEMIC INCLUSIVITY} To avoid paternalism (Bjorgvinsson, Ehn, \& Hillgren, 2010) and product superfluousness (Preece, Rogers, \& Sharp, 2002), the authorial team must be diverse and inclusive of potential users; the KOS, upon release, must be participatory via a publicly accessible feedback loop. The Map the Gap academic team strives to democratize the development and implementation process, enabling local community members to participate in design, influence the outcome, and eventually self-sustain the solution via structured co-design sessions.

The academic team recruits Philadelphia locals via flyers, announcements at civic association meetings, and discussions with community leaders. Locals are then welcomed to attend community engagement sessions wherein people engage in discussion, affinity clustering, card sorts, icon drawing, and visualizing the vote amongst other design research activities intended to promote community-efficacy, and yield viable information which informs the user-centered design of Map the Gap.

While traditional research studies involve only regimented engagement with participants, planning to learn from unstructured interactions with participants has helped the Map the Gap academic team to broaden its definition and pragmatic interpretation of the project's epistemic community; similarly, locals have become more adept at posing their knowledge in accessible ways to the academic team, thus creating a multidirectional communication pathway.

While unstructured participation, or irregular participation is often conceived of as the "unknown unknown" and is thus viewed as a threat (Zizek, 2006), or excuse to augment, sculpt, and proxy all non-conformist participation it must instead be interpreted as valid. Accordingly, the language of epistemic transposition must be adapted to be accessible to new voices who wish not to be translated but to be received in their raw form.

\section{Integrating Knowledge Transposition into Research De- sign}

Methods of obtaining viable information are largely dependent upon the way success of a KOS is measured (ie. with respect to who). Kitson et. al. attest to the need for knowledge translation strategies to be integrated methodologically with research design "from the outset" rather than tacked on linearly upon the collection of data (2013). In designing the qualitative research study which informs Map the Gap, the team was deliberate in structuring opportunities to feed community member feedback directly into the KOS itself.

During one focus group, community members were asked to individually arrange card decks into a visual map. Participants were able to add write-ins and subtract irrelevant cards as they saw necessary. They were then asked to draw icons for the categories they sorted the cards into. These icons and maps, upon synthesis (as opposed to interpretation, translation), will become the fundamental visual and conceptual organization of the Map the Gap KOS.

While the results of said activity were inevitably shaped by the values maintained by the academic team that designed the original card deck, future iterations of said deck will reflect adjustments and appropriations made by community members. In the aforementioned activity, both the academic team and local community members alike acted as participants, albeit at different temporal locations. The multidirectionality of influence in such a case is not to be treated as a tainted study, but rather as a reality of designing a KOS which requires a synergistic combination of efforts between people of diverse backgrounds. While ultimately the research team hopes to in-effect work itself out of a job by fostering a system that is entirely sustainable by the community of core users, such a vision will take time to achieve and there is recognizable value in early, conscientious collaboration that seeks to leverage the unique abilities of all parties via roles (rather than a hierarchy), and foster agency, autonomy, community efficacy, and community capacity.

\section{Opting for Roles Rather Than Hierarchies, Community Rather Than Dichotomies}

As aforementioned, the tensions of local/institutional collaborations require diverse epistemological communities to challenge academia's standards for expertise in order for practical applications of said local knowledge to be both communally useful and academically valid.

The process of navigating the aforementioned tensions is an iterative one riddled with reflection and reform. To reconcile evidence-hierarchy-related tensions, the Map the Gap 
team adopted a role-based organizational structure. The academic team now begins local engagement sessions with a disclaimer: "We are here to listen. We know that you are the experts on your community, and we ask humbly for a chance to learn from you." This disclaimer is a heartfelt and humble plea for a chance to live, learn, grow, and give collaboratively. It is critical to acknowledge our limited expertise with regards to the lived experience of local community members, and to seek consent for collaboration.

Preparing for a local engagement event, we have learned, involves willing oneself to be vulnerable by acknowledging the limits of one's expertise in the face of excessive and often unwarranted authorial capacity. Engagement is about learning to leverage one's privilege for power not to be voices for the oppressed, but to ensure that the oppressed have enduring seats at the table. Community engagement is about co-constructing common ground, identifying shared value, co-designing, compromising, reconciling, and realizing in an equitable, sustainable, empowering, and non-paternalistic way.

\section{CONCLUSION}

While we anticipate that the Map the Gap knowledge organization system will improve the lives of Philadelphians by helping mitigate housing insecurity, it can only do so given that a culture of health is promoted throughout the development process. As a team, we place great value in the process of collaboration we have engaged in over the past year. We hope that our collaboration will initiate a new era of campus-community relations (wherein "us" and "them" is re-defined) and provide all-around hope for a synergistic future. When we say "our project," we are not simply referring to a product produced by a team at Drexel University and the University of Pennsylvania, but a process in which two groups with historic strife came together to realize something of shared value.

As a team, we honed the plans for our knowledge organization system to address the realities of the West Philadelphia housing experience. What we originally thought to be necessary features of the interface are no longer the features we deem to be necessary. By identifying a shared mission of reducing the burden of housing insecurity, letting go of our notions about what the solution should look like, and conscientiously confronting the tensions associated with integrating epistemic and practical communities, we were able to be open minded in defining a strategy to realize our mission. Essentially, the social structure underlying the KOS permits such a fluidity of technical features, what we term the creation of a healthy sociotechnical infrastructure.

\section{Ethically Evocative Questions}

While it is presumable that the process of designing a knowledge organization system is void of emotion, it is, in our case, a highly emotive process of mending historic wounds between local community and institution. In all cases, however, it should be a pensive process. Whether acknowledged or not, there are politics and value judgements embedded in all artifacts (Winner, 1980). We must think of ourselves as both humanists and designers, institutionally motivated and yet autonomous agents inseparably, and thus will ourselves to consider the questions which invoke ethical assessments of our embodiments and the politics which result from the structures we channel our embodiments into. Such questions include: Who is advantaged by this work? Who is disadvantaged? What proxies are used in this work? What values are espoused in those proxies? Are those values conducive to the world I wish to see? To the world my neighbor wishes to see? Who will have access to this new resource? Who is building this technology and for who? Will variations in access fuel disparity? How can this technology be built and implemented to foster equity?

Then, we must address our findings boldly, and in unwavering, enduring, norm-challenging partnership.

\section{Use of this Case Study as a Template}

While we hope that the Map the Gap case study can be leveraged as a template for developing health KOS sociotechnical infrastructure, we advise that caution be exercised when transposing. Local history greatly informs the Map the Gap team's navigation of tensions. When strategizing for healthy KOS sociotechnical development, history should be localized as well (as opposed to explored generally). Additionally, it should be recognized that there is no such thing as a bad group; all peoples are capable of fruitful collaborations, albeit with trial and error. In coming to the conclusions discussed in this paper, the Map the Gap team experienced a range of successes and failures all of which encouraged further interation. Lastly, cultivating healthy KOS structures inevitably involves wealth redistribution (whether in the form of cultural capital, authority, privilege, etc.); thus it should be acknowledged that personal status is perhaps at stake but not endangered for with the creation of a healthy KOS sociotechnical structures comes a culture of health which emphasizes equitable well-being for all.

\section{Cultivating Inclusivity by Absolving Dichotomies}

If nothing else, this paper is a testament to the difficulties of describing dichotomy dissolution. It has been incredibly difficult to write about the formation of a diverse team in accessible terms without fragmenting the organism into its historic segments. As you adopt and grapple with the contents of this piece pragmatically, we encourage you to consider how best to communicate your practices of unification to the world.

\section{REFERENCES}

Abras, C., Maloney-Krichmar, D., \& Preece, J. (2004). Usercentered design. Bainbridge, W. Encyclopedia of HumanComputer Interaction. Thousand Oaks: Sage Publications 37(4), 445-456.

Atleo, E. R. (2004). Tsawalk: A Nuu-chah-nulth worldview. Vancouver: UBC Press.

Björgvinsson, E., Ehn, P., \& Hillgren, P. A. (2010, November). Participatory design and democratizing innovation. In Proceedings of the 11th Biennial participatory design conference (pp. 41-50). ACM.

Bjögvinsson, E., Ehn, P., \& Hillgren, P. A. (2012). Design things and design thinking: Contemporary participatory design challenges. Design Issues 28(3), 101-116.

Blumgart, J. (2018, January 31). City launches new program 
to help renters facing eviction. Retrieved from http://planphilly.com/articles/2018/01/31/city-launchesnew-program-to-help-renters-facing-eviction

Callon, M. (2004). The role of hybrid communities and socio-technical arrangements in the participatory design. Journal of the center for information studies 5(3), 3-10.

Castleden, H., Morgan, V. S., \& Lamb, C. (2012). "I spent the first year drinking tea": Exploring Canadian university researchers' perspectives on community-based participatory research involving Indigenous peoples. The Canadian Geographer/Le Géographe canadien, 56(2), 160-179.

Janack, M. (n.d.). Standpoint Epistemology Without the ?Standpoint??: An Examination of Epistemic Privilege and Epistemic Authority. Hypatia 12(2), 125-139.

Kitson, A., Powell, K., Hoon, E., Newbury, J., Wilson, A., \& Beilby, J. (2013). Knowledge translation within a population health study: how do you do it?. Implementation science $8(1), 54$.

Koedel, C. (2017, October 5). Examining faculty diversity at America's top public universities (Rep.). Retrieved https://www.brookings.edu/blog/brown-center-chalkboard/2017/10/05/examining-faculty-diversity-at-americas- top-public-universities/

Lavis, J. N., Robertson, D., Woodside, J. M., McLeod, C. B., \& Abelson, J. (2003). How can research organizations more effectively transfer research knowledge to decision makers?. The Milbank Quarterly 81(2), 221-248.

Preece, J., Rogers, Y., \& Sharp, H. (2015). Interaction design: Beyond human-computer interaction . New York, NY: John Wiley \& Sons.

Todd, P. A., \& Ladle, R. J. (2008). Hidden dangers of a 'citation culture'. Ethics in science and environmental politics 8(1), 13-16.

Washington, J., \& Wright, J. (2014, December 10). Protestors take over Gutmann's holiday party. The Daily Pennsylvanian. Retrieved from https://www.thedp.com/article/2014/12/protesters-take-over-gutmanns-holiday-party

Winner, L. (1980). Do Artifacts Have Politics? Daedalus, 109(1), 121-136. Retrieved from http://www.jstor.org/sta$\underline{\text { ble } / 20024652}$

Žižek, S. (2006). Philosophy, the "unknown knowns," and the public use of reason. Topoi 25(1-2), 137-142. doi:10.1007/s11245-006-0021-2 